\title{
Lepton Flavor Equilibration and Leptogenesis
}

\author{
D. Aristizabal Sierra, ,, Marta Losada,, , \\ ${ }^{1}$ INFN, Laboratori Nazionali di Frascati, C.P. 13, 100044 Frascati, Italy \\ ${ }^{2}$ Centro de Investigaciones, Universidad Antonio Nariño, \\ Cra 3 Este No 47A-15 Bloque 4, Bogotá, Colombia \\ ${ }^{3}$ Instituto de Física, Universidad de Antioquia, A.A.1226, Medellín, Colombia
}

\begin{abstract}
We study the role played in leptogenesis by the equilibration of lepton flavors, as could be induced in supersymmetric models by off diagonal soft breaking masses for the scalar lepton doublets $\tilde{m}_{\alpha \beta}$, or more generically by new sources of lepton flavor violation. We show that if $\tilde{m}_{\alpha \beta} \gtrsim 1 \mathrm{GeV}$ and leptogenesis occurs below $\sim 100 \mathrm{TeV}$, dynamical flavor effects are irrelevant and leptogenesis is correctly described by a one-flavor Boltzmann equation. We also discuss spectator effects in low scale leptogenesis by taking into account various chemical equilibrium conditions enforced by the reactions that are in thermal equilibrium. We write down the Boltzmann equation for low scale supersymmetric leptogenesis that includes flavor and spectator effects in the presence of lepton flavor equilibration, and we show how it reduces to a particularly simple form.
\end{abstract}

\section{INTRODUCTION}

Leptogenesis [1] is a theoretical mechanism that can explain the observed matter-antimatter asymmetry of the Universe. An initial lepton asymmetry is generated in the out-of-equilibrium decays of heavy singlet Majorana neutrinos, and is then partially converted in a baryon asymmetry by anomalous sphaleron interactions [2]. Heavy Majorana singlet neutrinos are also a fundamental ingredient of the seesaw model [3], that provides an elegant explanation of the suppression of neutrino masses with respect to all other Standard Model (SM) mass scales. Leptogenesis can be quantitatively successful without any fine-tuning of the seesaw parameters, and it is an intriguing 'coincidence' that a neutrino mass scale of the order of the atmospheric neutrino mass square difference is optimal for yielding the correct value of the baryon asymmetry. The possibility of giving an explanation of two apparently unrelated experimental facts (neutrino masses and the baryon asymmetry) within a single framework, makes the idea that baryogenesis occurred through leptogenesis very attractive.

The crucial role played by lepton flavor effects in leptogenesis was fully realized only a few years ago [4, 5, 6] (see [7, 8] for earlier studies of flavor effects in leptogenesis, and [9, 10] for recent reviews). Most extensions of the SM, and most noticeably among these, the Supersymmetric Standard Model (SSM), include new sources of Lepton Flavor Violation (LFV). The purpose of this letter is to show that if leptogenesis occurs at tempera- tures when these new sources mediate reactions that are in chemical equilibrium, then there are no flavor effects in leptogenesis. ${ }^{1}$ In particular, this is likely to happen in soft leptogenesis [11, 12, 13] that is a natural mechanism for low scale leptogenesis. In contrast, We include in our analysis also the effects of spectator processes [14, 15] that should be taken into account for a correct estimate of the final baryon asymmetry.

\section{LEPTON FLAVOR EQUILIBRATION}

With Lepton Flavor Equilibration (LFE) we refer to the effect of reactions that would bring the different lepton doublets $\ell_{\alpha}(\alpha=e, \mu, \tau)$ into chemical equilibrium. We will use as a general and most interesting example the SSM where, in the basis in which the charged lepton Yukawa couplings are diagonal, a source of LFV from soft supersymmetry breaking masses is generally present:

$$
\mathcal{L}_{\text {soft }} \supset \tilde{m}_{\alpha \beta}^{2} \tilde{\ell}_{\alpha}^{\dagger} \tilde{\ell}_{\beta}
$$

\footnotetext{
1 In this paper, we refer to "flavor effects" in the restricted sense of the dynamical effects that arise when the flavored CP asymmetries $\epsilon_{\alpha}$ are not proportional to the respective branching fractions for $N \rightarrow \ell_{\alpha}$ decays. In the (fine tuned) case when an exact proportionality exists, the final baryon asymmetry is simply enhanced by a factor corresponding to the number of lepton flavors that are in thermal equilibrium [5]. This result holds independently of the particular flavor configuration, it is unrelated to flavor dynamics, and is a simple consequence of fermion family replication. Thus, we include this effect in the general class of effects that, like the typical spectator effects, are unrelated to the dynamics of lepton flavors and of lepton flavor number violation.
} 
Here $\tilde{\ell}_{\alpha}$ are the superpartners of the $S U(2)$ lepton doublets. The terms in eq. (11) affect the flavor composition of the mass eigenstates, and as a result the $\ell_{\alpha} \tilde{\ell}_{\alpha}^{(\mathrm{int})} \tilde{G}$ vertex for the sleptons gauge eigenstates (where $\tilde{G}=\tilde{W}_{a}, \tilde{B}$ represent a $S U(2)$ or $U(1)$ gaugino) involve a unitary rotation to the slepton mass eigenstates:

$$
\tilde{\ell}_{\alpha}^{(\text {int })}=R_{\alpha \beta} \tilde{\ell}_{\beta}, \quad R_{\alpha \beta} \sim \delta_{\alpha \beta}+\mathcal{O}\left(\frac{\tilde{m}_{\alpha \beta}^{2}}{h_{\alpha}^{2} T^{2}}\right),
$$

where $h_{\alpha}>h_{\beta}$ is the relevant charged lepton Yukawa coupling that determines at leading order the (thermal) mass splittings of the sleptons.

The term in eq. (11) can induce fast LFV reactions, namely gaugino mediated $t$-channel processes $\ell_{\alpha} P \leftrightarrow$ $\tilde{\ell}_{\beta} \tilde{P}, \ell_{\alpha} \tilde{P} \leftrightarrow \tilde{\ell}_{\beta} P$, and $s$-channel processes $\ell_{\alpha} \tilde{\ell}_{\beta} \leftrightarrow P \tilde{P}$, with $P=\ell, Q, \phi_{u}, \phi_{d}$, where $Q$ and $\phi_{u, d}$ are the quark and Higgs doublets (for bino-reactions also the quark and leptons $S U(2)$-singlets $u, d, e$ contribute). For example, for the reduced cross section corresponding to $S U(2) t$ channel reactions we obtain:

$$
\begin{aligned}
\hat{\sigma}_{t}(s)= & R_{\alpha \beta} R_{\alpha \beta}^{*} \frac{g^{4}}{16 \pi} \Pi_{g} \times \\
& {\left[\left(2 \frac{m_{\tilde{W}}^{2}}{s}+1\right) \log \left(\frac{s+m_{\tilde{W}}^{2}}{m_{\tilde{W}}^{2}}\right)-2\right] }
\end{aligned}
$$

where $\Pi_{g} \equiv\left(\sum_{\beta \neq \alpha} g_{L_{\alpha}} g_{\tilde{L}_{\beta}}\right) \times \sum_{P} g_{P} g_{\tilde{P}}$ where $g_{L}, g_{P}, \ldots$ count the number of degrees of freedom (spin, isospin, color and flavor) of the corresponding particles, and $m_{\tilde{W}}^{2}=(9 / 2) g_{2}^{2} T^{2}$ is the $W$-ino thermal mass. Because of the several possible reactions and of the large number of degrees of freedom, $\Pi_{g}$ is a large number $\sim 10^{3}$. From eq. (3) and using eq. (2) (for $\alpha \neq \beta$ that is the case of interest) we can estimate the thermally averaged reaction density for $t$-channel $\alpha \leftrightarrow \beta$ transitions as:

$$
\begin{aligned}
\gamma_{t, \alpha \leftrightarrow \beta}= & \frac{T}{64 \pi^{4}} \int d s \sqrt{s} K_{1}\left(\frac{\sqrt{s}}{T}\right) \hat{\sigma}_{t}(s) \\
& \sim\left(\frac{g^{2} \tilde{m}_{\alpha \beta}^{2}}{h_{\alpha}^{2} T^{2}}\right)^{2} \frac{\Pi_{g} T^{4}}{2^{9} \pi^{5}} .
\end{aligned}
$$

Summing $s$-channel reactions and normalizing to the relativistic abundance of leptons we obtain the LFV rate

$$
\Gamma_{L F V} \sim \frac{\gamma_{\alpha \leftrightarrow \beta}}{T^{3} / \pi^{2}} \sim 10^{6} \frac{\tilde{m}_{\alpha \beta}^{4}}{T^{3}} .
$$

When this rate is faster than the Universe expansion $\Gamma_{H} \sim 25 T^{2} / M_{P}$ (with $M_{P}$ the Planck mass), asymmetries eventually present in the different lepton doublets equilibrate, meaning that their chemical potentials (that here and in the following are denoted with the same symbol than the corresponding particle) become equal: $\ell_{\alpha}=\ell$. According to eq. (5), this occurs roughly for

$$
T \lesssim 100\left(\frac{\tilde{m}_{\alpha \beta}}{1 \mathrm{GeV}}\right)^{4 / 5} \mathrm{TeV} .
$$

We see that even for moderate values of the off-diagonal soft breaking scalar masses, LFE is likely to be a generic feature in low scale supersymmetric leptogenesis.

\section{EQUILIBRIUM CONDITIONS}

To see what are the consequences of LFE on leptogenesis, we need to write the corresponding Boltzmann Equations (BE) taking into account all the chemical equilibrium conditions imposed by reactions that, at the specific temperature considered, are faster than the Universe expansion. Here we will concentrate on the temperature range $1 \mathrm{TeV} \lesssim T \lesssim 100 \mathrm{TeV}$, that is well above the electroweak phase transition, but low enough so that in the $\mathrm{SSM}$, LFE equilibration is likely to occur. In principle there are as many chemical potentials as there are particles in the thermal bath. However, a first set of conditions that are generally realized in the temperature range we are interested in allows to drastically reduce this number:

1. Since we will work at scales much higher than $M_{W}$, where total isospin $I_{3}$, hypercharge (and color) must be zero, gauge fields have vanishing chemical potential $W=B=g=0$ [16]. This also implies that all the particles belonging to the same $S U(3) \times S U(2) \times U(1)$ multiplet have the same chemical potential, that is, $\psi\left(I_{3}=+\frac{1}{2}\right)=\psi\left(I_{3}=\right.$ $\left.-\frac{1}{2}\right)$ for weak isospin, and similarly for color.

2. Chemical potentials for the three gauginos $\tilde{W}=$ $\tilde{B}=\tilde{g}$ are driven to zero once the supersymmetry breaking effects related to the Majorana soft gaugino masses $m_{1 / 2}$ attain chemical equilibrium [17], that is when $m_{1 / 2}^{2} / T \gtrsim \Gamma_{H}$. Note that since these 
rates are quadratic in $m_{1 / 2}$ while the corresponding rates for LFE go as $\tilde{m}_{\alpha \beta}^{4}$ in studying LFE it is certainly reasonable to assume $\tilde{W}=\tilde{B}=\tilde{g}=0$. In turn, the vanishing of the gaugino chemical potentials implies that particles within the same supermultiplet have the same chemical potential $\tilde{\psi}=\psi$.

3. Similarly to what happens for the gauginos, the Higgsino mass term $\mu_{\phi_{u, d}} \phi_{u} \phi_{d}$ ensures that $\phi_{d}+$ $\phi_{u}=0$. We then denote $\phi_{u}=-\phi_{d}=\phi$.

4. Because of generation-mixing interactions, we take generation-independent quark potentials $Q_{i}=Q$, $u_{i}=u$ and $d_{i}=d$. Fast LFV interactions yield $(\alpha \neq \beta)$

$$
\ell_{\alpha}+P=\tilde{\ell}_{\beta}+\tilde{P}=\ell_{\beta}+P
$$

where $P$ represents, for example, any one of the $\ell, Q, \phi_{u, d} S U(2)$ doublets, and the second equality follows from condition 2 . This yields $\ell_{\alpha}=\ell_{\beta}=\ell$, that is the chemical potential of the lepton doublets are also generation independent.

5. Given that $\ell_{\mu}=\ell_{\tau}$, the Yukawa couplings interactions for the right handed $\mu$ and $\tau$ leptons, that are both in equilibrium below $\left(1+\tan ^{2} \beta\right) \times 10^{9} \mathrm{GeV}$, yield $\tau=\mu$. As regards the right handed electron, its Yukawa interaction attains equilibrium below $T_{e} \sim 20-100 \mathrm{TeV}$ [18], depending also on the value of $\tan \beta .^{2}$ If also the off diagonal mass terms for the right handed sleptons $\tilde{m}_{e \alpha}^{R}(\alpha=\mu, \tau)$ are particularly suppressed, then the right handed (s)electrons would remain out of chemical equilibrium in an interesting range above $T_{e}$. We thus leave open the possibility that $\mu \neq e=0$.

\footnotetext{
${ }^{2}$ Reactions mediated by the small up-quark Yukawa coupling $Y_{u}$ will be in equilibrium at temperatures below $T_{u} \sim\left(Y_{u} / Y_{e}\right)^{2} T_{e} \approx$ $\left(m_{u}^{2} / m_{e}^{2} \tan ^{2} \beta\right) T_{e}$. Thus, if $\tan \beta<m_{u} / m_{e} \approx \mathcal{O}(10)$, reactions mediated by $Y_{u}$ will always be in equilibrium when the electron Yukawa interactions are in equilibrium. If $\tan \beta \gg m_{u} / m_{e}$, the up-quark Yukawa interactions can remain out-of-equilibrium in most of the temperature regime we are considering. This would yield slightly different numerical results.
}

Because of the previous conditions, we are left with six independent chemical potentials: $Q, u, d, \ell, \mu$ and $\phi$. They should satisfy the following additional conditions:

Yukawa couplings equilibration:

$$
\begin{aligned}
Q-u+\phi_{u} & =0, \\
Q-d+\phi_{d} & =0, \\
\ell-\mu+\phi_{d} & =0 .
\end{aligned}
$$

Electroweak sphalerons equilibrium (QCD sphalerons equilibrium do not impose further constraints [15]):

$$
3 Q+\ell=0
$$

Hypercharge neutrality:

$$
3(Q+2 u-d-\ell)-2 \mu-e+\phi_{u}-\phi_{d}=0,
$$

where, depending on the temperature range, $e=\mu$ or $e=0$. These are five conditions, and therefore all the chemical potentials can be expressed in terms of just one, that we choose to be $\ell$. The solution for $e=\mu$ reads:

$$
Q=-\frac{\ell}{3} ; u=\frac{5 \ell}{21} ; d=-\frac{19 \ell}{21} ; \mu=\frac{3 \ell}{7} ; \phi=\frac{4 \ell}{7},
$$

with minor numerical changes in $u, d, \mu, \phi$ when $e=0$. We can now use these equations to express $\ell$ in terms of the asymmetry in the $B-L$ charge, that is the relevant quantity for writing the $\mathrm{BE}$ in our temperature regime since it is not violated by EW sphalerons. We denote the number density asymmetry for a particle $p$ normalized to the entropy density $s$ as $Y_{\Delta p}=\left(n_{p}-n_{\bar{p}}\right) / s$, and $Y_{\Delta p}$ normalized to the equilibrium density $Y_{p}^{\mathrm{eq}}$ is denoted as $y_{\Delta p}=Y_{\Delta p} / Y_{p}^{\mathrm{eq}}$. Let us also remember that, because of boson (B)/fermion (F) statistics, the relation between chemical potentials and particle density asymmetries reads $y_{\Delta B} / y_{\Delta F}=2 \mu_{B} / \mu_{F}$. Assuming that all effects of particle masses can be neglected [19], and depending if the right-handed electron is or is not in chemical equilibrium, then we have:

$$
\begin{aligned}
& \frac{Y_{\Delta_{B-L}}^{(e=\mu)}}{Y_{\Delta \ell}}=\frac{9}{\ell}[2 Q+u+d-(2 \ell+\mu)]=-\frac{237}{7}, \\
& \frac{Y_{\Delta_{B-L}}^{(e=0)}}{Y_{\Delta \ell}}=\frac{9}{\ell}\left[2 Q+u+d-\left(2 \ell+\frac{2}{3} \mu\right)\right]=-\frac{426}{13} .
\end{aligned}
$$




\section{THE BOLTZMANN EQUATIONS}

When accounting for flavor effects is a mandatory condition $\left(T \lesssim 10^{12} \mathrm{GeV}\right.$ [4, 5, 7]) one should write a set of three $\mathrm{BE}$, one for each of the conserved flavor charges $\Delta_{\alpha}=B / 3-L_{\alpha}$. To illustrate the importance of LFE, here we write simplified BE including only decays and inverse decays. Also, we write just the BE for the heavy Majorana neutrinos, the effects of LFE on the equations for the sneutrinos are completely analogous. The BE for the evolution of the heavy neutrino density as usual reads: $-\dot{Y}_{N}=\left(y_{N}-1\right) \gamma_{D}$ where $\gamma_{D}$ is the total $N$ decay rate into $\ell, \phi_{u}$ and their superpartners, $y_{N}=Y_{N} / Y_{N}^{\text {eq }}$ and the time derivative is $\dot{Y}_{N}=s H z d Y_{N} / d z$, with $z=M_{N} / T$. By taking equal decay rates into particles and superpartners $\gamma_{D_{\ell}} \simeq \gamma_{D_{\tilde{\ell}}} \simeq \gamma_{D} / 2$ we can write the evolution equation for the flavor charges as:

$$
\begin{aligned}
-\dot{Y}_{\Delta_{\alpha}} & =\left(y_{N}-1\right) \epsilon_{\alpha} \gamma_{D} \\
- & \frac{1}{2}\left(y_{\Delta \ell}+y_{\Delta \phi}+y_{\Delta \tilde{\ell}}+y_{\Delta \tilde{\phi}}\right) B_{\alpha}\left(\frac{1}{2} \gamma_{D}\right) \\
= & \left(y_{N}-1\right) \epsilon_{\alpha} \gamma_{D}-\frac{1}{2}\left(\frac{33}{7} y_{\Delta \ell}\right) B_{\alpha}\left(\frac{1}{2} \gamma_{D}\right)
\end{aligned}
$$

where $\epsilon_{\alpha}$ and $B_{\alpha}$ are respectively the CP asymmetry and the branching fraction for the decay $N \rightarrow \ell_{\alpha}+\tilde{\ell}_{\alpha}$. In the last line we have used $y_{\Delta \phi}=\frac{8}{7} y_{\Delta \ell}, y_{\Delta \tilde{\phi}}=\frac{4}{7} y_{\Delta \ell}$ and $y_{\Delta \tilde{\ell}}=2 y_{\Delta \ell}$. Now in order to integrate eq. (16), one should express the asymmetry density $y_{\Delta \ell}$ that is weighting the strength of the washouts, in terms of the charge densities $y_{\Delta_{\alpha}}$. The important thing to notice at this point is that, regardless of the details of the resulting expression, as a consequence of LFE the washout weights do not carry any flavor index. We can thus readily sum up the three flavored equations and obtain

$$
-\dot{Y}_{\Delta_{B-L}}=\left(y_{N}-1\right) \epsilon \gamma_{D}+\frac{1}{4}\left(\frac{11}{79}\right) y_{\Delta_{B-L}} \gamma_{D}
$$

where $\epsilon=\sum_{\alpha} \epsilon_{\alpha}$ and eq. (14) has been used. For the case in which the right handed electron is out of equilibrium, eq. (15) together with $y_{\Delta \phi}=2 y_{\Delta \tilde{\phi}}=\frac{14}{13} y_{\Delta \ell}$ should be used instead, and the washout numerical coefficient changes slightly: $\frac{11}{79} \rightarrow \frac{10}{71}$.

A few remarks are now in order:

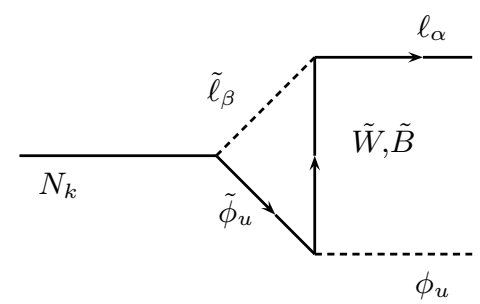

FIG. 1: Vertex diagram generating lepton flavor violating $\mathrm{CP}$ asymmetries in the decays of the heavy Majorana neutrinos $N_{k} \rightarrow \ell_{\alpha} \phi_{u}$. Similar diagrams appear for $N_{k} \rightarrow \tilde{\ell}_{\alpha} \tilde{\phi}_{u}$ and in the decays of the neutrinos superpartners $\tilde{N}_{k} \rightarrow \ell_{\alpha} \tilde{\phi}_{u}, \tilde{\ell}_{\alpha} \phi_{u}$.

1. LFE allows to recast the BE equation in just one equation for $B-L$, thus killing effectively flavor effects. Recalling that in the temperature regime $T \lesssim 10^{9} \mathrm{GeV}$ also all effects of the heavier neutrinos $N_{2,3}$ are absent [20], we can conclude that the one-flavor BE eq. (17) gives a complete description of the dynamics of leptogenesis. Note that such a drastic simplification depends just on the hypothesis of flavor equilibration, and it applies in particular to soft leptogenesis as long as $\tilde{m}_{\alpha \beta} \gtrsim 1 \mathrm{GeV}$ and $M_{N} \lesssim 100 \mathrm{TeV}$. In case $\tilde{m}_{\alpha \beta}$ is diagonal to an extremely good approximation, then flavor effects can survive, ${ }^{3}$ and can have important consequences also for soft leptogenesis [21].

2. Purely Flavored Leptogenesis (PFL) models [22], that are defined by the condition $\sum_{\alpha} \epsilon_{\alpha}=0$, are unable to generate a baryon asymmetry in the presence of LFE since the source term in the BE eq. (17) vanishes. ${ }^{4}$ In this respect, it is interesting to note that the same LFV soft masses responsible for LFE also generate lepton flavor violating CP asymmetries through interference of the tree level decay amplitude with the loop diagram depicted in fig. 1 . The CP asymmetry in the decay of the $N_{k}$ seesaw neutrino into SM particles induced by this diagram

\footnotetext{
3 For flavor effects to kick back in it is in fact sufficient to have e.g., $\tilde{m}_{e \alpha} \approx 0$ for $\alpha=\mu, \tau$. Below $T_{e}$ also $\tilde{m}_{e \alpha}^{R} \approx 0$ is required.

4 In the PFL model studied in [22] LFE does not occur, because LFV is coupled to heavy messengers states with mass several times larger than $M_{N}$, and thus it is strongly suppressed.
} 
reads:

$\epsilon_{\ell_{\alpha} \phi_{u}}^{N_{k}}=-\frac{3 g^{2}+g^{\prime 2}}{8 \pi\left(\lambda^{\dagger} \lambda\right)_{k k}} \sum_{\beta} \operatorname{Im}\left(\frac{\lambda_{\beta k}^{*} \tilde{m}_{\beta \alpha}^{2} \lambda_{\alpha k}}{M_{N_{k}}^{2}}\right)$,

and similar expressions hold for the other flavored CP asymmetries $\epsilon_{\tilde{\ell}_{\alpha} \tilde{\phi}}^{N_{k}}, \epsilon_{\tilde{\ell}_{\alpha} \phi}^{\tilde{N}_{k}}$, and $\epsilon_{\ell_{\alpha} \tilde{\phi}}^{\tilde{N}_{k}}$. From eq. (18) it is readily seen that $\sum_{\alpha} \epsilon_{\ell_{\alpha} \phi_{u}}^{N_{k}}=0$. In the absence of LFE, this condition by itself would not impede to generate a lepton asymmetry [5, 22] and, rather interestingly, the decoupling of the $\mathrm{CP}$ asymmetries (enhanced as $\left.\sim g^{2}\right)$ from the washouts $\left(\propto \lambda^{2}\right)$ would have yielded a new mechanism for low scale leptogenesis and for avoiding the gravitino problem. However, to obtain sufficiently large CP asymmetries one must require $\tilde{m}_{\alpha \beta}^{2} / M_{N}^{2} \gtrsim 10^{-6}$, and this unavoidably implies LFE. We can thus conclude that the flavored CP asymmetries eq. (18) are irrelevant to leptogenesis.

\section{CONCLUSIONS}

Flavor effects can produce large enhancements of the baryon asymmetry yield of leptogenesis. If the CP asymmetry in one lepton flavor is particularly large (and note that it could even be larger than the total lepton $\mathrm{CP}$ asymmetry) and at the same time the associated washouts are suppressed by a small branching fraction for $N$ decays to that flavor, the baryon asymmetry will be sizeably larger than what would be obtained in a oneflavor approximation based on the total lepton CP asymmetry and on the total washout rates.

The effectiveness of flavor effects relies, however, on the condition that the dynamics of the different lepton flavors remains sufficiently decoupled during the leptogenesis era. In this paper we have shown that when LFV interactions are sufficiently fast to equilibrate the asymmetries in the different lepton doublets, flavor effects disappear. In this situation, the dynamics of leptogenesis can be again described correctly by means of a single $\mathrm{BE}$ for the evolution of B-L.

It is well known that in the SM+seesaw, leptogenesis remains a high energy mechanism, and that to lower the leptogenesis scale, say below $\sim 10^{8} \mathrm{GeV}$, physics be- yond the $\mathrm{SM}+$ seesaw is required. However, new sources of LFV are a quite common features in models for new physics, and thus when leptogenesis is embedded within new physics models, it is important to verify if flavor effects are relevant or not in that particular realization.

In this paper, we have illustrated this point by studying the SSM+seesaw, that probably is the most interesting example. The SSM allows to produce a baryon asymmetry at a scale as low as a few $\mathrm{TeVs}$, through the mechanism of soft leptogenesis. However, the SSM also includes new sources of LFV from the supersymmetry soft breaking sector, and we have shown that if the offdiagonal soft masses for the scalar lepton doublets are larger than about $1 \mathrm{GeV}$, then there are no flavor effects in soft leptogenesis, as long as it occurs below roughly $T \sim 100 \mathrm{TeV}$. We conclude that in the presence of LFE, a simple and qualitatively correct description of leptogenesis can be given in terms of just one equation for the evolution of the lepton asymmetry. However, for a numerically accurate estimate, an equation for the evolution of $B-L$ (like eq. (17)) that takes into account electroweak sphalerons equilibrium as well as the effects of other spectator processes, is required.

\section{Acknowledgments}

We thank S. Davidson for illuminating conversations during the early stages of this work, and M.C. GonzalezGarcia for several remarks on the preliminary draft. M.L. acknowledges the hospitality of IPN-Lyon, LNF-Frascati and IFT-Madrid during the completion of this work. M.L. was supported by the Ecos-Nord program. The work of M.L. and E.N. is supported in part by Colciencias under contract number 1115-333-18739.

* Electronic address: diego.aristizabal@lnf.infn.it

† Electronic address: malosada@uan.edu.co

‡ Electronic address: enrico.nardi@lnf.infn.it

[1] M. Fukugita and T. Yanagida, Phys. Lett. B 174, 45 (1986).

[2] V. A. Kuzmin, V. A. Rubakov and M. E. Shaposhnikov, Phys. Lett. B 155, 36 (1985). 
[3] P. Minkowski, Phys. Lett. B 67421 (1977); T. Yanagida, in Proc. of Workshop on Unified Theory and Baryon number in the Universe, eds. O. Sawada and A. Sugamoto, KEK, Tsukuba, (1979) p.95; M. Gell-Mann, P. Ramond and R. Slansky, in Supergravity, eds P. van Niewenhuizen and D. Z. Freedman (North Holland, Amsterdam 1980) p.315; P. Ramond, Sanibel talk, retroprinted as hep-ph/9809459; S. L. Glashow, in Quarks and Leptons, Cargèse lectures, eds M. Lévy, (Plenum, 1980, New York) p. 707; R. N. Mohapatra and G. Senjanović, Phys. Rev. Lett. 44, 912 (1980).

[4] A. Abada, S. Davidson, F. X. Josse-Michaux, M. Losada and A. Riotto, JCAP 0604 (2006) 004 arXiv:hep-ph/0601083.

[5] E. Nardi, Y. Nir, E. Roulet and J. Racker, JHEP 0601 (2006) 164 arXiv:hep-ph/0601084.

[6] A. Abada, S. Davidson, A. Ibarra, F. X. Josse-Michaux, M. Losada and A. Riotto, JHEP 0609 (2006) 010 arXiv:hep-ph/0605281.

[7] R. Barbieri, P. Creminelli, A. Strumia and N. Tetradis, Nucl. Phys. B 575, 61 (2000) (for the updated version of this paper see arXiv:hep-ph/9911315.).

[8] T. Endoh, T. Morozumi and Z. h. Xiong, Prog. Theor. Phys. 111, 123 (2004) arXiv:hep-ph/0308276; T. Fujihara, S. Kaneko, S. Kang, D. Kimura, T. Morozumi and M. Tanimoto, Phys. Rev. D 72, 016006 (2005) arXiv:hep-ph/0505076.

[9] S. Davidson, E. Nardi and Y. Nir, Phys. Rept. 466, 105 (2008); arXiv:0802.2962 [hep-ph].

[10] A. Strumia, arXiv:hep-ph/0608347 E. Nardi, arXiv:hep-ph/0702033 Y. Nir, arXiv:hep-ph/0702199 M. C. Chen, arXiv:hep-ph/0703087; E. Nardi, arXiv:0706.0487 [hep-ph]; A. Pilaftsis, arXiv:0904.1182 [hep-ph].

[11] Y. Grossman, T. Kashti, Y. Nir and E. Roulet, Phys. Rev. Lett. 91, 251801 (2003) arXiv:hep-ph/0307081;
[12] G. D'Ambrosio, G. F. Giudice and M. Raidal, Phys. Lett. B 575, 75 (2003) arXiv:hep-ph/0308031;

[13] Y. Grossman, T. Kashti, Y. Nir and E. Roulet, JHEP 0411, 080 (2004) arXiv:hep-ph/0407063.

[14] W. Buchmuller and M. Plumacher, Phys. Lett. B 511, 74 (2001) arXiv:hep-ph/0104189.

[15] E. Nardi, Y. Nir, J. Racker and E. Roulet, JHEP 0601, 068 (2006) arXiv:hep-ph/0512052.

[16] S. Y. Khlebnikov and M. E. Shaposhnikov, Nucl. Phys. B 308, 885 (1988). J. A. Harvey and M. S. Turner, Phys. Rev. D 42, 3344 (1990). H. K. Dreiner and G. G. Ross, Nucl. Phys. B 410, 188 (1993).

[17] L. E. Ibanez and F. Quevedo, Phys. Lett. B 283, 261 (1992) arXiv:hep-ph/9204205; T. Inui, T. Ichihara, Y. Mimura and N. Sakai, Phys. Lett. B 325, 392 (1994) arXiv:hep-ph/9310268.

[18] J. M. Cline, K. Kainulainen and K. A. Olive, Phys. Rev. Lett. 71, 2372 (1993) arXiv:hep-ph/9304321; J. M. Cline, K. Kainulainen and K. A. Olive, Phys. Rev. D 49, 6394 (1994) arXiv:hep-ph/9401208.

[19] V. A. Rubakov and M. E. Shaposhnikov, Usp. Fiz. Nauk 166, 493 (1996) [Phys. Usp. 39, 461 (1996)] arXiv:hep-ph/9603208; D. J. H. Chung, B. Garbrecht and S. Tulin, JCAP 0903, 008 (2009) arXiv:0807.2283 [hep-ph]].

[20] G. Engelhard, Y. Grossman, E. Nardi and Y. Nir, Phys. Rev. Lett. 99, 081802 (2007) arXiv:hep-ph/0612187.

[21] C. S. Fong and M. C. Gonzalez-Garcia, JHEP 0806, 076 (2008) arXiv:0804.4471 [hep-ph]]; C. S. Fong and M. C. Gonzalez-Garcia, JHEP 0903, 073 (2009) arXiv:0901.0008 [hep-ph]].

[22] D. Aristizabal Sierra, M. Losada and E. Nardi, Phys. Lett. B 659, 328 (2008) arXiv:0705.1489 [hep-ph]]; D. A. Sierra, L. A. Munoz and E. Nardi, arXiv:0904.3043 [hep-ph]; arXiv:0904.3052 [hep-ph]. 